\title{
Issues in Internet Radio
}

Yasushi Ichikawa, Kensuke Arakawa, Keisuke Wano, and Yuko Murayama

Dept. of Software and Information Science, Iwate Prefectural University

152-52 Sugo, Takizawa, Takizawa-mura, Iwate,Japan

\{ichikawa, araken\}@comm.soft.iwate-pu.ac.jp,

g031x169@edu.soft.iwate-pu.ac.jp, murayama@iwate-pu.ac.jp

\begin{abstract}
The World-wide Web(WWW) works now as the infrastructure over the Internet for multimedia applications. Internet radio is one of those applications and its growth is explosive. We have started operating an Internet Radio station with streaming music services since April 2000. An Internet radio can broadcast music over the network regardless of such geographic restrictions as the traditional radio systems have. There are some problems and services due to the Internet. This paper reports our operation as well as the issues. We propose our idea on some novel radio services as well.
\end{abstract}

\section{Introduction}

During the 80's the question was for what exact applications the Internet would be used best. We now know that the answer is WWW. The Internet has grown dramatically since WWW was introduced in the end of the 80's. Indeed, WWW is considered now as the infrastructure over the Internet for multimedia applications.

Internet radio is one of those applications. The growth of the number of Internet radio stations is explosive. There are more than 5000 stations operating over the Internet. The number of Internet radio stations has been increasing about 1000 stations each year.

The purpose of our research is to identify the issues to be dealt with by Internet Radio. This paper reports our initial effort to set up a radio station as well as its operation for several months. We describe issues and present an idea of some novel radio services.

\section{Internet Radio Systems}

The Internet radio stations are classified into two types according to their operations; commercial ones and private ones. The private stations operate differently from the commercial ones including the traditional radio broadcast stations. The private ones would select the music more from the service provider's viewpoint, whereas commercial ones need to provide the music favored possibly by many listeners. A famous commercial station would keep having more than 200 listeners.

E. Gregori et al. (Eds.): NETWORKING 2002, LNCS 2345, pp. 1129-1134 2002.

(C) Springer-Verlag Berlin Heidelberg 2002 
An Internet radio system has a client-server structure. A radio station has a server, and a user needs to have a client system such as an MP3 player. The multimedia authoring tools and the Internet have made it possible for us to set up private radio stations easily.

There are two types of music streaming services available on the Internet. One is to download music data and then play it. The other is to play music on music streaming server on a real-time basis. Our radio station uses the latter.

There are three systems available for setting up an Internet radio system, viz. The Real system [2], Shoutcast [3], and Icecast [4]. Real System is a server for a specific client system, the Realplayer. Most of the Internet radio stations use Real System. Icecast and Shoutcast provide MP3 streaming servers. MP3 is an MPEG Audio Layer 3, a compression format [5].

\section{$3 \quad$ Flip over Radio(FOR)}

In this research we set up our own radio station on the Internet called "Flip Over Radio (FOR)." At the moment we operate FOR on an experimental and private basis. We broadcast Indie music which is made originally by unknown artists who work independently from record companies. They have a limited opportunity in publishing their music such that the listeners can obtain the information only from specific magazines and music stores in Japan.

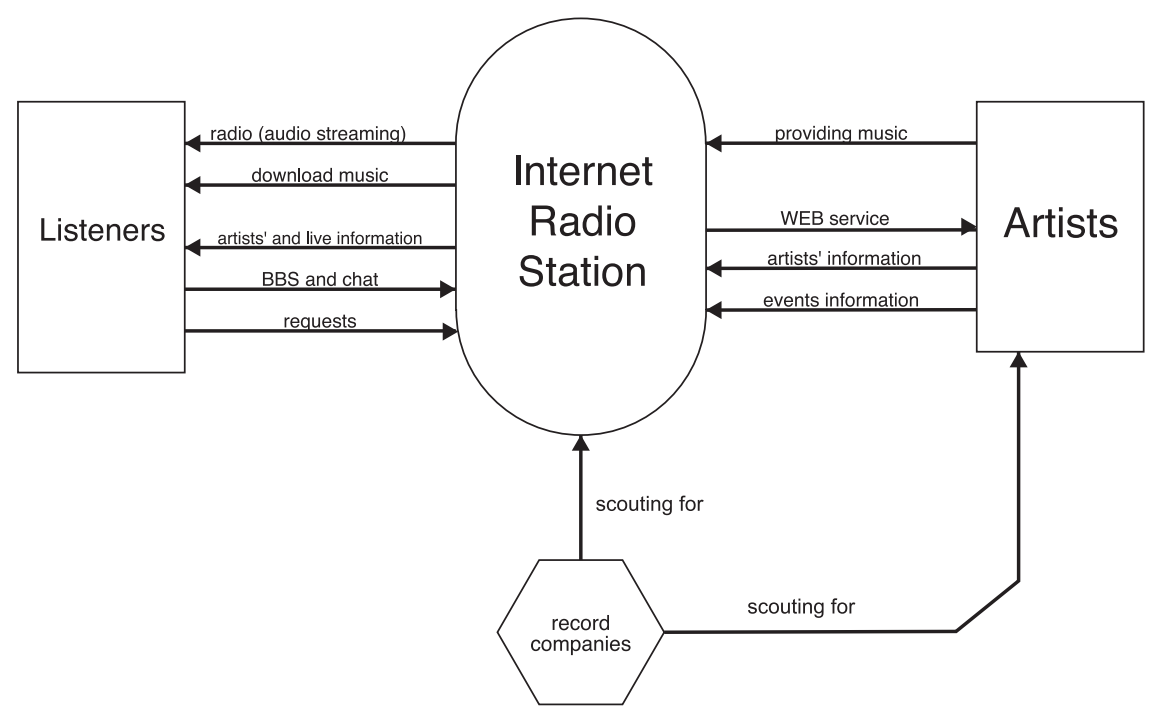

Fig. 1. The operational model of FOR

Fig. 1 shows the model of our radio operation. Our Internet radio station provide such an opportunity for both artists and listeners to exchange the in- 
formation on music and artists. Our radio site is a media for this exchange. The artists provide the music that they composed and played as well as the related information. We provide them with tools such as the one to make their home page as well as the message board so that they can communicate with the listeners. Commercial promoters could make use of the information we provide to find a new artist and music, so that an artist could have an opportunity to get a commercial contract.

Table 1 shows the configuration of the server system of FOR.

Table 1. The server system of FOR

\begin{tabular}{|c|l|}
\hline CPU & AMD K6-2 400MHz (Over DriveProcessor) \\
\hline MEMORY & $48 \mathrm{M}$ \\
\hline OS & Laser 5 Linux 6.0 \\
\hline HTTPserver & Apache \\
\hline Streaming & Icecast $[\underline{4}$ \\
Application & $\begin{array}{l}\text { Shout } \\
\text { Icedj }\end{array}$ \\
& Liveice \\
& {$[7]$} \\
\hline
\end{tabular}

Icecast is used to broadcast music. Shout selects a music to broadcast, and passes the music data to Icecast. Liveice is a real-time re-encoder and passes the encoded data to Icecast. We can mix several MP3 streams and audio inputs from mic(microphone) and Liveice. Icedj is used to run an Icecast radio station such that broadcasts a music at a certain time as scheduled in a program. It can be used together with Icecast to show the information on music being broadcast on the radio station's WWW page.

\section{The Operation Report}

We have been operating FOR since April 2000. Fig. 2 shows the number of total user access per month. We have not had so many users, presumably it is not because of Indie music, but due to poor amount of contents. Users would not listen to an Internet radio station if it broadcast the same songs repeatedly.

During July and August in 2001, we revised icecast in the latest version, so that the facility of the registration function started working well, which registers our radio server to the access ranking server on the Internet. 3 percent of connections were from our university, and 20 percent of connections were from Japan.

We found two requirements. One was that a user needs an easy-to-use interface. The other was that a lot of contents are required. We may well need a user interface in JAVA Applet, so that the software is installed automatically. A station with poor contents would not have the users who would visit the radio station site repeatedly. 


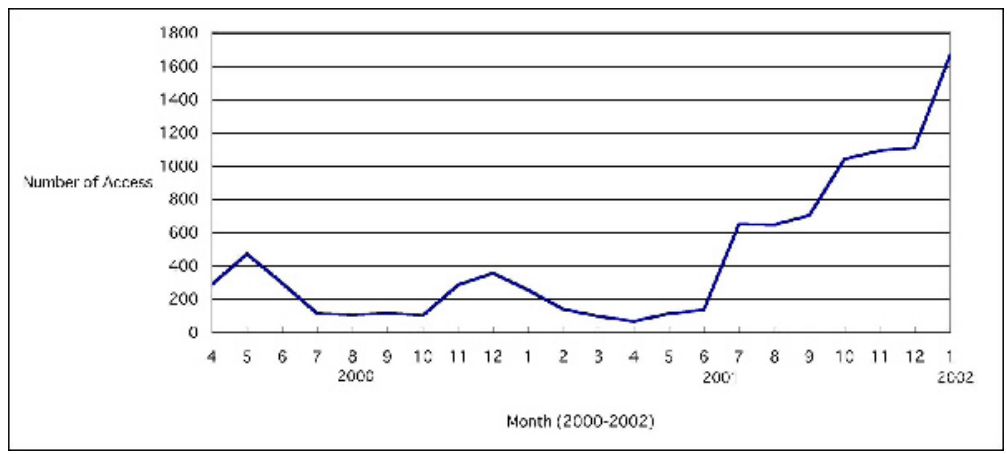

Fig. 2. User access per month (2000 - 2002)

\section{User's Private Channel}

We are planning to provide users with private channels, so that users can listen to their favorite music. This novel type of service is only possible on the Internet, but not on the traditional radio systems.

Fig. 3 shows the design of a private channel. The private channel operations are as follows :

1. A user registers his/her desirable channel ID and favorite music information on the web page and receives from the server a URL.

2. The registration process sends the registered information to a database engine which selects music. The database engine makes the play list of the user's favorite music.

3. The channel making process makes the user's private channel with the channel ID and the play list. The user makes access to the URL and listen to the music with the MP3 player.

We have implemented the first part, and half of the third part, from the above list. For the second part, we are planning to construct the music database with some attributes such as quiet and noisy, so that user's favorite music tunes can be selected automatically according to the user's taste.

There is a problems with this service. If we provide users with private channels on demand, we will require to run as many private channel processes as the number of user requests. The more private channel processes we have, the more loads the server gets and the slower the system operation becomes. We may need to explore the tradeoff between the performance of the server and the number of private channels. We may well need dynamic channel management.

\section{Distributed Streaming}

If we provide our radio service from only one site, the server site will be a bottle neck as the number of users increases. We propose distributed streaming 


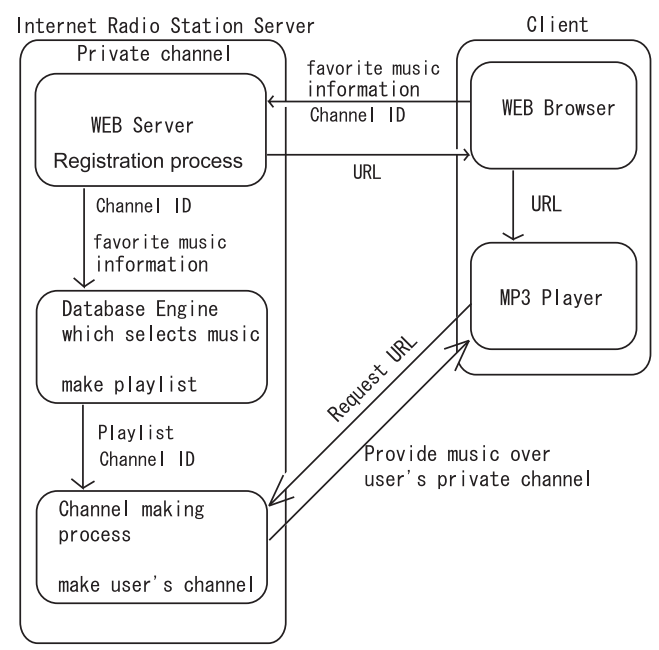

Fig. 3. The design of a private channel

by setting up relay servers. A relay server is an application level router which forwards MP3 data stream. A radio station transmits single music data to a relay server. A user connects to the nearest relay server. The relay server makes copies of the music data and sends them down to users. This operation looks similar to multicast as in Resource ReSerVation Protocol (RSVP) 8 whose multicast function operates at the network level.

Content Delivery and Distribution Networks(CDNs) may be one of a possible tool for this 9], CDNs provide users with an access to one of the distributed servers in the different locations over the Internet. The servers have a cache of an original content. A user has an access to the nearest CDNs server. There are many products and services of CDNs. We need further investigation on the use of CDNs for the Internet radio service.

We plan to provide users with private channels by making use of CDNs according to user's taste from the user's nearest relay server. Firstly, the relay server caches the music contents of the original server. Secondly, the private channel server makes a play list according to a request from a user, and sends it to the user's nearest relay server. Thirdly, its relay server makes channel and provides music according to users' taste with the play list.

\section{Related Work}

Most of the radio stations are operating on a commercial basis.

Among them the following site is one of those that have many services and users: http://www.netradio.com. It has more than 100 channels. They are classified firstly into global categories such as pop, rock, and so on. In a category, the channels are classified further into subcategories such as chronological groups. 
The commercial sites provide users with a shopping function as well so that users can purchase CDs of their favorite music.

Another radio station: http://www.wolffm.com. deals with the various types of streaming such as MP3 streaming Realplayer, and Windows Media Player.

Our radio station provides only MP3 streaming and 32kbps bit-rate at the moment, however, we are planning to provide some other bit-rates of MP3 as well in future. Our radio station is managed on a private and non-profitable basis with one channel, the contents of 150 music tunes, and some artists information at the moment. We are providing only with the specific type of copyright-free music and the information on the almost unknown artists in Japan.

\section{Conclusion}

This paper introduced the Internet radio from the viewpoint of a service provider. Internet radio systems have many different properties from the traditional radio system. For example, a cultural revolution could be possible in music, since any type of music could be delivered over the Internet and some of them would never appear on the traditional commercial media.

We reported on the operation of our Internet radio station and identify some issues to be dealt with in future. The issues include providing users with their private channels and making the delivery system distributed.

Future work includes examining the database engine function of the private channel, making the delivery service distributed, and providing an easier user interface. We plan to implement those required functions into a client system using JAVA Applet with Java Media Frame(JMF) [10, provided in the Multimedia library of JAVA.

\section{References}

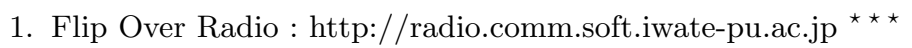

2. Real system : http://www.realnetworks.com/ ${ }^{\star \star \star}$

3. Shoutcast : http://www.shoutcast.com ***

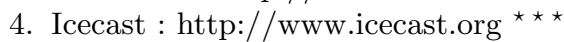

5. Fraunhofer Research : http://www.iis.fhg.de/amm/ ***

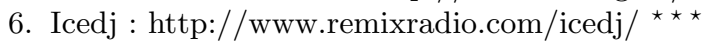

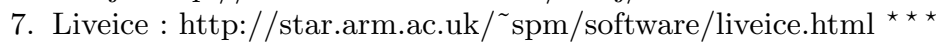

8. L. Zhang, S. Deering, D. Estrin, S. Shenker, and D. Zappala: RSVP: A New Resource ReSerVation Protocol, IEEE Network Vol.7 Issue 5 pp.8-18 (Sep. 1993)

9. Balachander Krishnamurthy, Craig Wills and Yin Zhang, On the Use and Performance of Content Distribution Networks, ACM SIGCOMM Internet Measurement Workshop 2001

10. JMF : http://www.java.sun.com/products/jave-media/index.html *** 